\title{
TIC no Ensino Fundamental: quando, onde e como estão sendo publicadas as pesquisas nesse segmento
}

\author{
Lívia Anselmo Maciel $^{1}$, Clevi Rapkiewicz, Rute Vera Maria Favero ${ }^{1}$ \\ ${ }^{1}$ Colégio de Aplicação - Universidade Federal do Rio Grande do Sul (UFRGS) \\ \{livia.anselmo13, clevi.capufrgs\}@gmail.com, rute@ufrgs.br
}

\begin{abstract}
The primary education target audience is digital natives who, however, do not always make use of Information and Communication Technologies (ICT) in the teaching and learning process. Understanding the state of the art of computer science research in this segment of education is important in order to propose innovations in both the technological and pedagogical spheres. In this context, this article presents a Systematic Review of Literature (SRL) considering two congresses and three journals in the area of informatics in education in Brazil in the interval of years (2012-2016). It can be observed that there is an evolution in the amount of research although there is concentration in the final years and concentration in certain regions of the country.
\end{abstract}

Resumo: $O$ ensino fundamental envolve nativos digitais que, no entanto, nem sempre fazem uso de Tecnologias de Informação e Comunicação (TIC) no processo de ensino e aprendizagem. Compreender como se encontra a pesquisa de informática na educação voltada para esse segmento da educação é importante para propor inovações, tanto no âmbito tecnológic, quanto pedagógico. Nesse contexto, este artigo apresenta uma Revisão Sistemática de Literatura (RSL), considerando dois congressos e três revistas da área de Informática na Educação, no Brasil, no intervalo de cinco anos (2012-2016). Pode-se observar que há uma evolução na quantidade de pesquisas ainda que haja concentração nos anos finais e em certas regiões do país.

\section{Introdução}

No sistema de educação brasileiro, o ensino fundamental tem duração de nove anos. É a etapa mais longa da Educação Básica e, na modalidade regular, atende estudantes entre 6 e 14 anos. O ensino fundamental também é ofertado na modalidade de Educação de Jovens e Adultos (EJA). Por ser um longo período, com constantes mudanças na realidade de cada aluno, há uma imposição de desafios na elaboração de currículos para essa etapa de escolarização.

Em pesquisa realizada pelo Centro Regional de Estudos para Desenvolvimento da Sociedade da Informação (CETIC, 2015) com alunos do ensino fundamental, anos iniciais e finais, $75 \%$ declararam ficar mais interessados nas aulas com o uso de TIC.

Os Parâmetros Curriculares Nacionais (BRASIL, 1997), do Ministério da Educação (MEC), tratam como indiscutível a necessidade crescente do uso de computadores pelos alunos como instrumento de aprendizagem escolar, para que possam estar atualizados em relação às tecnologias da informação e comunicação, não apenas para questões de lazer e sociais, mas também educativas. Esses nativos digitais (usando aqui o termo cunhado por Prensky, 2001), público-alvo desse segmento de ensino, é influenciado pela tecnologia de tal maneira que afeta a forma como eles 
pensam e se comportam, mas se trata de uma via de mão dupla - a maneira como as crianças pensam e se comportam está influenciando e moldando a própria internet e as tecnologias digitais.

Segundo a Base Nacional Comum Curricular (MEC, 2017) compete a educação básica a utilização de TIC de forma crítica, significativa, reflexiva e ética nas diversas práticas do cotidiano (incluindo as escolares) ao se comunicar, acessar e disseminar informações, produzir conhecimentos e resolver problemas.

Gutiérrez (2016) lembra que tecnologia, informação e comunicação estão presentes no ensino fundamental por uma necessidade como quando aparecem na criação de jogos educativos, em ambientes virtuais, para melhorar trabalhos em equipes e no processo de aprendizagem de programação para crianças, por exemplo. No entanto, essa necessidade e benefício da inserção de TICs na educação escolar surge acompanhada de impactos diretos na relação ensino-aprendizagem-tecnologia que precisam ser notados.

Com base nas demandas apontadas pelos PCN e pela BNCC no uso de TIC no ensino fundamental é importante identificar o estado da arte da Informática na Educação nesse segmento de ensino, no Brasil. Elencar o que tem sido feito pode permitir apontar novos caminhos para pesquisas focadas em aspectos tecnológicos e, também, pode permitir a proposição de ações de inovação pedagógica integrando TIC nas práticas dos professores e nos cursos de formação de professores, seja formação inicial (licenciaturas) ou continuada.

Para tanto, este artigo está organizado em 4 seções, além da presente introdução. $\mathrm{Na}$ seção 2 apresentamos brevemente algumas revisões sistemáticas da literatura presentes em revistas e congressos de informática na educação, no Brasil, indicando, ao mesmo tempo, que revisões sistemáticas têm estado presentes, mas não especificamente por segmento de ensino. Na seção 3, apresentamos a metodologia utilizada. A análise dos resultados aparece na seção 4, enquanto, na seção 5, apresentamos algumas considerações finais sobre o que foi exposto.

\section{Revisões sistemáticas de literatura na área de informática na educação}

Nos anos recentes, tem sido recorrente a presença de revisões sistemáticas de literatura, através da metodologia RSL ou outras, nos principais eventos nacionais de Informática na Educação e nas principais revistas da área. Pesquisa realizada em dois eventos e em três revistas, sintetizada na Tabela 1, usando os descritores 'revisão+literatura', 'revisão+bibliografia', 'revisão+bibliográfica', 'RSL', 'revisão sistemática' apontam predominância de revisões sistemáticas voltadas para aspectos técnicos da informática.

Tabela 1: RSL em Informática na Educação - 5 anos

\begin{tabular}{|c|c|c|c|c|c|c|}
\hline Ano/Veículo & RBIE & RENOTE & IETP & SBIE & WIE & Total \\
\hline 2012 & 0 & 2 & 1 & 7 & 0 & 10 \\
\hline 2013 & 1 & 4 & 1 & 5 & 1 & 12 \\
\hline 2014 & 1 & 1 & 0 & 5 & 0 & 7 \\
\hline 2015 & 4 & 2 & 0 & 19 & 2 & 27 \\
\hline 2016 & 3 & 9 & 1 & 16 & 1 & 30 \\
\hline Total & 9 & 18 & 3 & 52 & 4 & 86 \\
\hline
\end{tabular}

Já na Revista Novas Tecnologias na Educação (RENOTE), entre os 18 artigos recuperados, apenas uma se refere ao ensino fundamental. Mas o tema analisado na revisão feita (MEDEIROS e SCHIMIGUEL, 2012) é específico sobre jogos educativos. 
Na Revista de Informática na Educação Teoria e Prática (IETP), o uso dos descritores apontados resulta na recuperação de 18 artigos; no entanto, apenas 3 são de revisão sistemática. Somente um deles era voltado para o público, em certa medida, de nativos digitais, em que ressaltava o uso da fotografia como estratégia metodológica em pesquisas com crianças (SILVA, 2016).

No que concerne ao Simpósio Brasileiro de Informática na Educação (SBIE) verifica-se ser este o veículo que mais concentra artigos de revisão. No entanto, nenhum dos 52 localizados nos anos recentes tem foco por segmento de ensino.

No Workshop de Informática na Escola (WIE), a consulta, considerando os resultados dos últimos cinco anos, resultou em 4 artigos, sendo que, em um deles, há uma breve revisão do uso de TIC em escolas de educação infantil (BERGAMASCO e BERGAMASCO, 2013).

Com base na análise das revisões bibliográficas encontradas, verifica-se a pertinência de apresentar este estudo para a compreensão do estado da arte da Informática na Educação, neste segmento de ensino voltado para nativos digitais.

\section{Metodologia}

Para conseguir identificar o que está sendo produzido, onde está sendo publicado, quando e onde estão sendo divulgadas as produções acadêmicas que tratam do uso de TIC no ensino fundamental, optou-se por uma Revisão Sistemática de Literatura, ou apenas RSL. Segundo Biolchini et al (2005), RSL é uma técnica de pesquisa baseada em evidências da literatura científica, conduzida formalmente, seguindo fases de um protocolo bem definidas. São elas: realização de atividades de planejamento; execução e sumarização dos resultados; respostas às questões de pesquisa definidas.

Para análise foram considerados artigos publicados na Revista Brasileira de Informática na Educação (RBIE), na Revista Novas Tecnologias na Educação (RENOTE) e a Informática na Educação: Teoria e Prática (IETP). Somam-se aqui também as consultas aos anais do Simpósio Brasileiro de Informática na Educação (SBIE) e do Workshop de Informática na Escola (WIE). A delimitação temporal foi de 5 anos (2012-2016) uma vez que, considerando-se a rápida evolução tecnológica na área de informática, recuperar pesquisas mais antigas sem haver um propósito de resgate histórico ou construção de uma linha do tempo não se aplica ao estudo sendo feito.

A localização dos artigos que interessavam a este estudo foi feita por meio de consulta a edições anteriores das publicações delimitando a busca para incluir apenas aquilo que contivesse, em seu resumo, título ou palavras-chave as expressões ensino fundamental e educação básica. Quando as produções não continham as palavras chaves procuradas, mas continham, por exemplo, conteúdos específicos do ensino fundamental, foi necessária uma análise dos resultados, discussões e metodologias para identificar se estavam de acordo com a busca efetuada.

Ao final do levantamento quantitativo, realizado manualmente e organizado em tabelas, localizaram-se 84 publicações de interesse e que contemplavam a temática escolhida para a RSL. A partir disso, trabalhou-se na busca da compreensão do estado da arte da pesquisa recente ( 5 anos) em Informática na Educação tendo como guia as perguntas sintetizadas na Tabela 2 . 
VI Congresso Brasileiro de Informática na Educação (CBIE 2017)

Anais do XXIII Workshop de Informática na Escola (WIE 2017)

Tabela 2 : Perguntas norteadoras da revisão sistemática

1. Qual o total de artigos destinados ao Ensino Fundamental durante esses cinco anos?

2. Quais os veículos de publicação que deram espaço ao Ensino Fundamental durante os últimos 5 anos?

3. Dentro do Ensino Fundamental, quais os segmentos que estão sendo contemplados com essa produção acadêmica?

4. Aonde no Brasil estão sendo realizados estes estudos?

Outras perguntas visando identificar plataformas de hardware e software utilizadas, bem como estratégias pedagógicas aplicadas, também foram utilizadas, buscando aproximar a análise de um estudo do estado da arte da pesquisa em Informática na Educação no ensino fundamental, porém, por questão de limitação de espaço, não são abordadas no presente artigo.

\section{Resultados e Discussão}

Esta seção está organizada em 4 subseções, expostas de acordo com as perguntas norteadoras do estudo apresentado.

\subsection{A evolução temporal}

Do total de artigos publicados, mais de dois mil, em todas as edições da RBIE, RENOTE, IETP, SBIE e WIE nos últimos cinco anos, foram encontradas apenas 84 produções acadêmicas que focam nas TIC no âmbito do ensino fundamental (Figura 1).

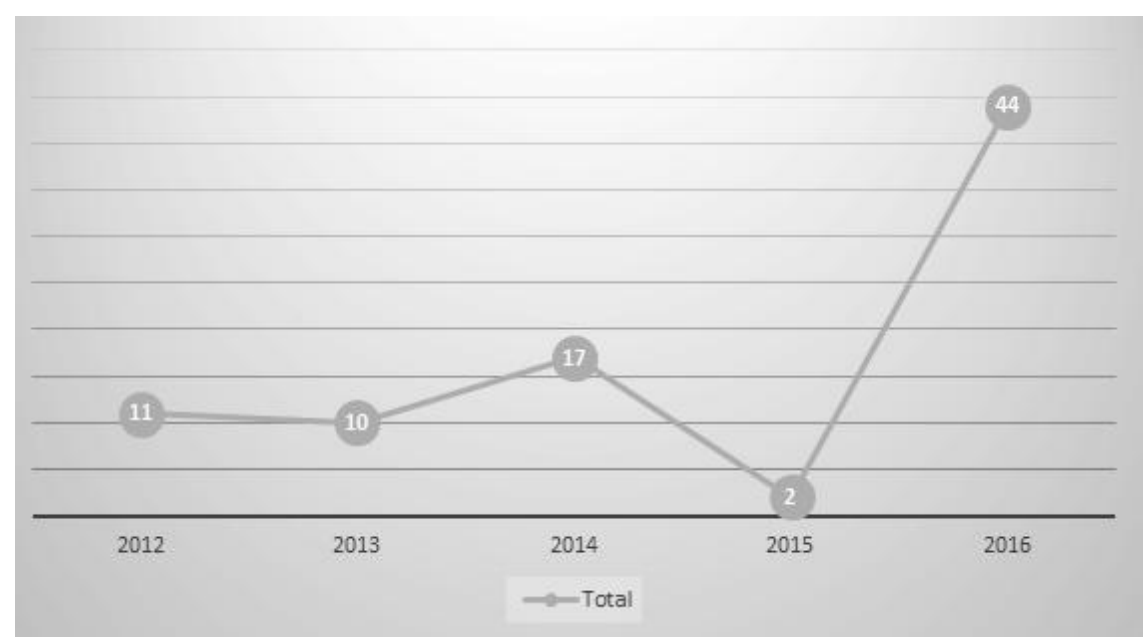

Figura 1: Total de artigos que falam de TIC no ensino fundamental, no período de 5 anos

Conforme apontado na Figura 1, é possível constatar que, nos anos de 2012 e 2013, as produções acadêmicas voltadas ao ensino fundamental nas publicações em que se propõe analisar esse artigo permanecem estagnadas, variando de 11 a 10 respectivamente. Em 2014, esse quadro muda e passa a ser ampliado com 17 produções publicadas. No entanto, em 2015 o número que, pelo contexto histórico da época, tenderia a crescer, caiu significativamente, havendo apenas 2 artigos. Pelos dados, podese afirmar que, 2016, foi o auge da produção e publicação acadêmica sobre TIC no ensino fundamental, quando se atingiu 44 trabalhos divulgados para a sociedade, nos 
veículos analisados. Seria de se esperar que 2016 fosse um ano em que houvesse uma maior aplicação das TIC em sala de aula, como pode-se ver no Relatório Mundial de 2016 - Dividendos Digitais (BANCO MUNDIAL, 2016), a partir do momento em que são oferecidas mais inovações tecnológicas (TSUKAMOTO, FIALHO e TORRES, 2014). O relatório salienta que as tecnologias digitais geram dividendos digitais e promovem o desenvolvimento, permitindo que mais pessoas tenham acesso a estas tecnologias, possibilitando o aumento da inclusão digital.

\subsection{Veículos de publicação que mais deram destaque}

De todas as publicações e eventos, a que mais publicou sobre TIC no ensino fundamental foi o WIE, o que é coerente com o objetivo principal do evento, qual seja, a divulgação de iniciativas nacionais de aplicação de TIC. Foram 31 trabalhos publicados. Em seguida, com 23, aparece a RENOTE. O SBIE, com ênfase mais em desenvolvimento do que o WIE, contribuiu com 18 produções, o que pode ser considerado baixo, levando-se em conta o período de 5 anos, podendo significar o pouco interesse dos pesquisadores em focar em tecnologias para o ensino fundamental. Entre as publicações que menos deram espaço para os trabalhos que destacam a inserção de TIC no ensino fundamental estão a RBIE com 7, e a IETP com 5, conforme é possível ver na Figura 2.

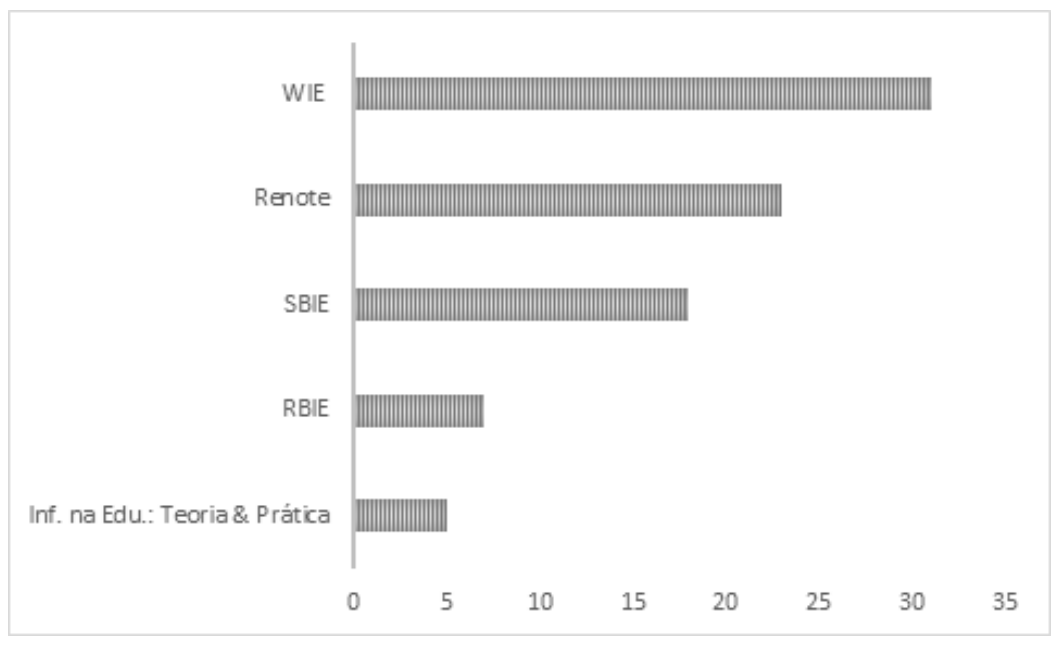

Figura 2: Veículos de publicação que mais deram destaque

\subsection{Os segmentos contemplados}

Considerando-se que o ensino fundamental de 9 anos é a etapa mais longa do sistema formal da educação básica brasileira, a análise diferenciou o segmento dos anos iniciais $\left(1^{\circ}\right.$ ao $5^{\circ}$ ano) do $2^{\circ}$ segmento referente a tudo que inclui os anos finais ( $6^{\circ}$ ao $9^{\circ}$ ano). Quando o artigo envolvia ambos os segmentos, ao invés de somar duas vezes a publicação (uma vez em cada segmento), optou-se por incluir essa categoria específica. Destacou-se também artigos que contemplavam a formação de professores do ensino fundamental (Figura 3). Com isso, foi possível notar que a prioridade dos trabalhos publicados nesse intervalo de tempo tem sido o segundo segmento, cujo levantamento apontou 38 publicações. O primeiro segmento aparece logo em seguida, com 22 trabalhos publicados. Alguns trabalhos, no entanto, contemplam os dois segmentos e somam um total de 16. Exclusivamente para a formação de professores do ensino fundamental, foram encontrados 6 trabalhos, o que sugere que possa estar ocorrendo uma ampliação da pesquisa relativa à formação de recursos humanos para atuar com 
TIC nesse segmento. Dois trabalhos contemplam tanto a formação de professores, assim como os dois segmentos.

O aumento do uso das TIC, principalmente, das Mídias Sociais, em cursos de nível superior é bem notório (FAVERO, 2016), enquanto o uso no ensino fundamental vem mostrando um crescimento, ainda, lento, mas, como aponta Couto Junior (2013), "as práticas pedagógicas mediadas pelos artefatos tecnológicos atraem mais a atenção das crianças, e um dos desafios levantados a serem enfrentados é a maior promoção entre a cultura escolar e as práticas culturais das crianças com as mídias".

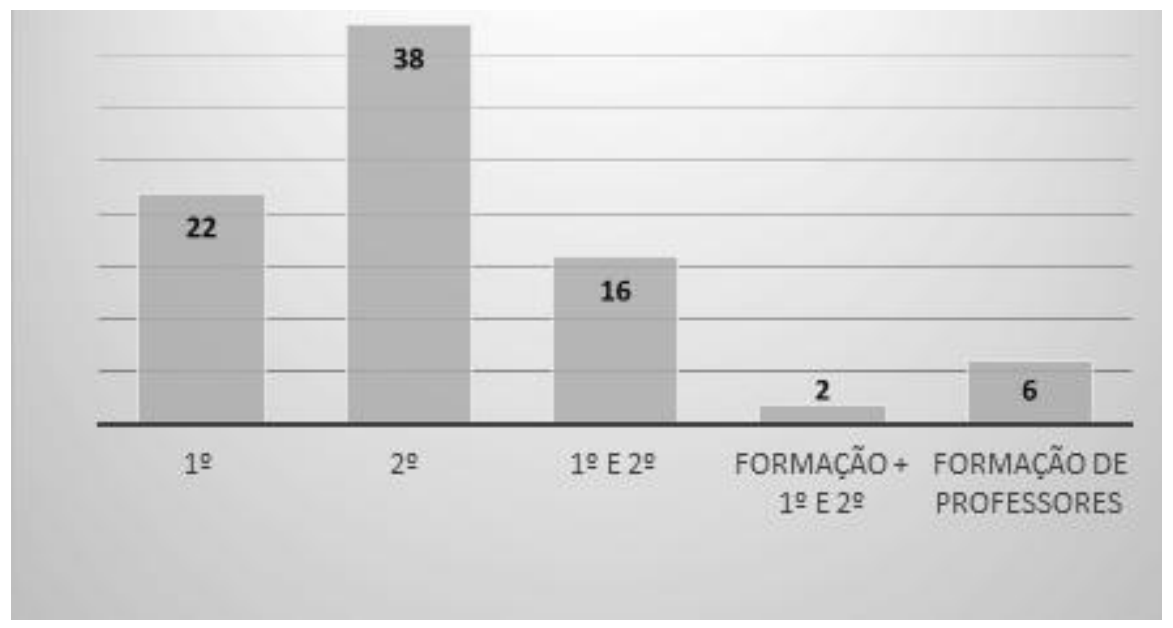

Figura 3: Total de artigos publicados por segmento.

\subsection{As regiões onde se concentram as pesquisas}

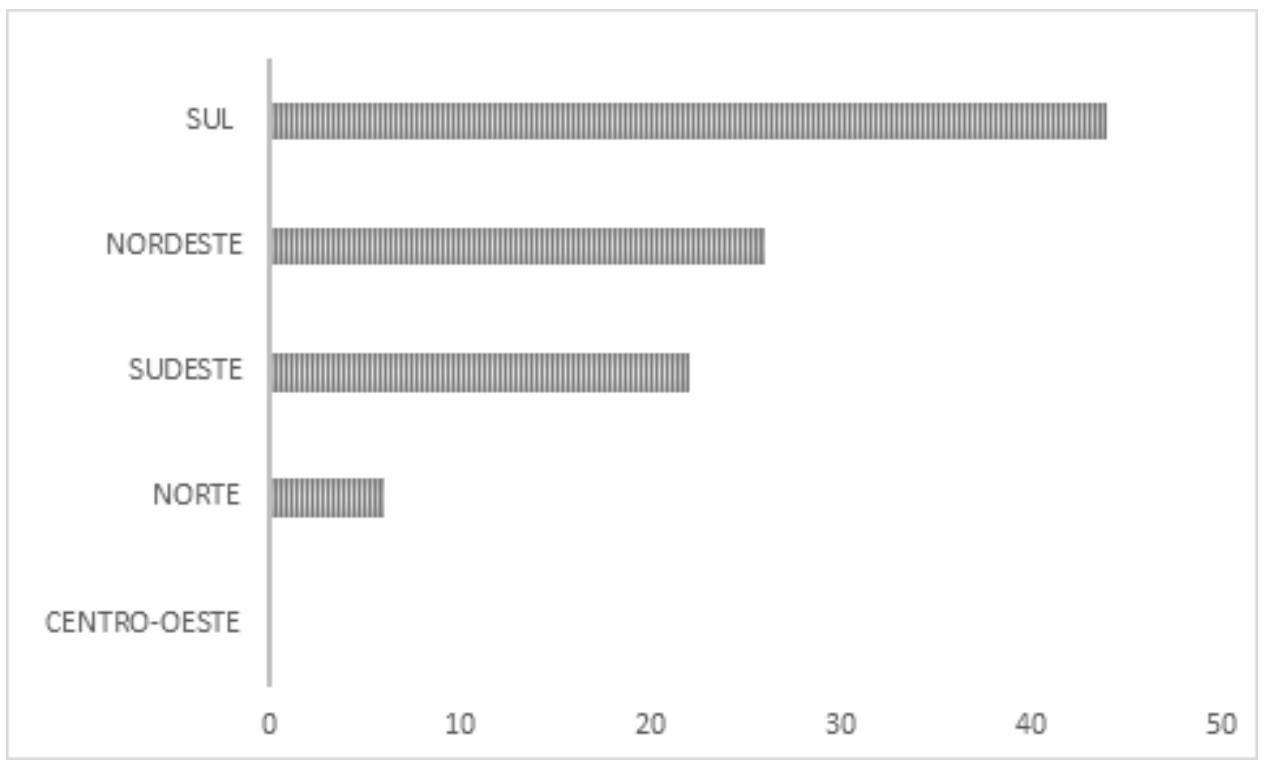

Figura 4: Total de artigos TIC por região.

Os artigos encontrados nesta RSL estão distribuídos em 4 regiões (Figura 4); com maior concentração no Sul, seguida da região Nordeste, as quais respondem por $78 \%$ das produções. Apenas a região centro-oeste não possui instituições que produziram conteúdo para publicação. A região Nordeste, conforme o levantamento, contribui com 26 artigos científicos divididos entre os 9 estados que a compõem. No Sudeste, foram 22 produções, colocando a região como a terceira maior produtora de trabalhos dessa área. Por último, a região Norte, teve apenas 6 artigos e pesquisas 
divulgados. Essa concentração da pesquisa em Informática na Educação, para esse segmento, é convergente com a concentração de universidades que atuam na pesquisa, no Brasil, nas três regiões. Poder-se-ia pensar em políticas públicas de incentivo à pesquisa diferenciada, conforme as realidades locais das diferentes regiões.

\section{Considerações finais}

Após o levantamento e identificação de conteúdos que tratam da inserção de TIC no ensino fundamental, nos últimos cinco anos, aplicando-se a metodologia RSL, foi possível concluir que, nesse período, foram publicados 84 artigos, pesquisas e relatos de experiências. Nesse total, os anais do WIE foram os que mais deram espaço para esses trabalhos. O segundo segmento $\left(6^{\circ}\right.$ ao $9^{\circ}$ ano) é o que mais recebeu estudos, com 38 trabalhos e a região Sul do país é onde mais se produziram pesquisas.

Nesse processo, entre as dificuldades encontradas esteve a necessidade de realizar todas as pesquisas manualmente, devido aos mecanismos de busca serem limitados e não conseguirem finalizar as buscas. Além disso, os descritores utilizados nesta pesquisa eram amplos e dificultaram a localização de trabalhos que pudessem servir para esse levantamento. Algumas publicações, não continham nos títulos, nem nas palavras-chaves, nem em seus resumos as expressões ensino fundamental $e$ educação básica ou qualquer expressão que pudesse identificar ser desse segmento, exigindo assim uma busca manual mais profunda nos trabalhos.

Partindo do acima exposto, é pertinente repetir a pergunta feita pelo pesquisador Pireddu (2014, p. 14): "Por que as tecnologias de informação e de comunicação [...] continuam a ser escassamente utilizadas, diminuídas ou ignoradas dos sistemas educativos institucionais?", Sabendo-se que estas tecnologias oferecem tantas possibilidades, dentre elas informação e conhecimento, é importante que as pesquisas continuem; assim como essa pesquisa pode ser ampliada para uma continuidade que permita responder questões que incluam entender a forma como a inserção de TIC tem sido realizada, quais as disciplinas que mais têm explorado esses recursos, quais os recursos de hardware e software mais utilizados, quais as áreas da computação mais exploradas para esse segmento. Há também a possibilidade de levar essas questões em uma pesquisa que permita identificar como as TIC são inseridas no ensino infantil e médio, nesses últimos cinco anos. Também é importante lembrar que as publicações analisadas foram da área de informática e tecnologia e que existe a possibilidade de que mais e novos trabalhos estejam em publicações da área da Educação.

\section{Referências}

BANCO MUNDIAL. Relatório sobre o Desenvolvimento Mundial de 2016: Dividendos Digitais. Overview booklet. Banco Mundial, Washington, D.C. Licença: Creative Commons Attribution CC BY 3.0 IGO. 2016.

BERGAMASCO, Elizabeth Carneiro; BERGAMASCO, Cristina Carneiro. A utilização das Tecnologias de Informação e Comunicação na Educação Infantil: avanços e desafios. XIX Workshop de Informática na Escola (wie 2013), Campinas, Sp, v. 0, n. 0, p.0-0, nov. 2013. Disponível em: <http://zip.net/bxtMhG〉. Acesso em: 25 jun. 2017.

BIOLChInI, J., MiAN, P. G., NATALI, A. C. C., TRAVASSOS, G. H. Systematic Reviews in Software Engineering. Technical Report ES 679/05, 31 p. 2005. 
VI Congresso Brasileiro de Informática na Educação (CBIE 2017)

Anais do XXIII Workshop de Informática na Escola (WIE 2017)

BRASIL. Secretaria de Educação Fundamental. Parâmetros curriculares nacionais: introdução aos parâmetros curriculares nacionais / Secretaria de Educação Fundamental. - Brasília: MEC/SEF, 1997. Disponível em: 〈http://zip.net/brk1Qp〉. Acesso em: 07 de junho de 2017.

CETIC. TIC Educação, 2015. Pesquisa sobre o uso das Tecnologias de Informação e Comunicação nas escolas brasileiras. Disponível em: < ttp://zip.net/bdtLYH>. Acesso em: 06 de maio de 2017.

COUTO JUNIOR, Dilton Ribeiro. Mídias e Educação Infantil: desafios na prática pedagógica. Informática na Educação: teoria e prática, Porto Alegre, v. 16, n. 2, p. 131-146, jul./dez. 2013.

FAVERO, Rute Vera Maria. A cultura dos usos das redes na academia: um olhar de professores universitários, brasileiros e italianos, sobre o uso das mídias sociais na docência. Disponível em: <http://www.lume.ufrgs.br/handle/10183/151639>. Acesso em: 26 de junho de 2017.

MEC. Base Nacional Comum Curricular. $3^{\text {a }}$ edição, 2017. Disponível em: <http://basenacionalcomum.mec.gov.br/images/pdf/1_BNCC-Final_Introducao.pdf >. Acesso em 07 de junho de 2017.

MEDEIROS, Maxwell de Oliveira; SCHIMIGUEL, Juliano. UMA ABORDAGEM PARA AVALIAÇÃO DE JOGOS EDUCATIVOS: ÊNFASE NO ENSINO FUNDAMENTAL. Revista Novas Tecnologias na Educação - Renote, Porto Alegre, Rs, v. 10, p.0-0, dez. 2012. Disponível em: <http://zip.net/bbtLkZ>. Acesso em: 25 jun. 2017.

PIREDDU, Mario. Social learning: le forme comunicative dell'apprendimento. Milano: Guerini Scientifica, 2014.

PRENSKY, M. Digital Native, digital immmigrants. Digital Native immigrants. On the horizon, MCB University Press, Vol. 9, N.5, October, 2001. Disponível em: <http://zip.net/bcmMtF>. Acesso em: 30 de junho de 2017.

SILVA, Paula Marques da. O uso da fotografia como estratégia metodológica em pesquisas com crianças: uma revisão de literatura. Informática na Educação: Teoria \& Prática, Porto Alegre, v. 19, dez. 2016. Disponível em: <http://zip.net/bbtLkY>. Acesso em: 25 de maio de 2017.

TSUKAMOTO, N. M. S.; FIALHO, N. N. e TORRES, P.L. A face educacional do Facebook: um relato de experiência. 2014. In: PORTO, C.; SANTOS, E. Orgs). Facebook e educação: publicar, curtir, compartilhar. Campina Grande, EDUEPB, 2014, pp.349-364. Disponível em: <http://books.scielo.org/id/c3h5q/pdf/porto9788578792831-20.pdf>. Acesso em: 26 de julho de 2017.

\subsection{Referência do Corpus ${ }^{1}$}

CONGRESSO BRASILEIRO DE INFORMÁTICA NA EDUCAÇÃO - CBIE. 2012, Rio de Janeiro, RJ. Anais do XVIII Workshop de Informática na Escola (WIE 2012). Rio de Janeiro, RJ. Sociedade Brasileira de Computação - SBC, 2012. Disponível em: <http://www.br-ie.org/pub/index.php/wie/issue/view/52>. Acesso em: 23 de junho de 2017.

\footnotetext{
${ }^{1}$ Considerando-se a limitação de páginas, optou-se por referenciar os anais e números completos nos quais houve a localização de um ou mais artigos.
} 
CONGRESSO BRASILEIRO DE INFORMÁTICA NA EDUCAÇÃO - CBIE. 2013, Campinas, SP. Anais do XIX Workshop de Informática na Escola (WIE 2013). Campinas, SP. Sociedade Brasileira de Computação - SBC, 2013. Disponível em: <http://www.br-ie.org/pub/index.php/wie/issue/view/80>. Acesso em: 23 de junho de 2017.

III CONGRESSO BRASILEIRO DE INFORMÁTICA NA EDUCAÇÃO - CBIE. 2014, Dourados, MS. Anais do XX Workshop de Informática na Escola (WIE 2014). Dourados, MS: Sociedade Brasileira de Computação - SBC, 2014. Disponível em: <http://www.br-ie.org/pub/index.php/wie/issue/view/88>. Acesso em: 23 de junho de 2017.

V CONGRESSO BRASILEIRO DE INFORMÁTICA NA EDUCAÇÃO - CBIE. 2016, Uberlândia, MG. Anais do XXII Workshop de Informática na Escola (WIE 2016). Uberlândia, MG: Sociedade Brasileira de Computação - SBC, 2016. Disponível em: <http://www.br-ie.org/pub/index.php/wie/issue/view/156>. Acesso em: 23 de junho de 2017.

CONGRESSO BRASILEIRO DE INFORMÁTICA NA EDUCAÇÃO - CBIE, 2013, Campinas, SP. 24 Simpósio Brasileiro de Informática na Educação - SBIE 2013 Campinas, SP: Sociedade Brasileira de Computação - SBC, 2014. Disponível em: <http://www.br-ie.org/pub/index.php/sbie/issue/view/78>. Acesso em: 23 jun. 2017.

III CONGRESSO BRASILEIRO DE INFORMÁTICA NA EDUCAÇÃO - CBIE, 2014, Dourados, MS. $\mathbf{2 5}^{\circ}$ Simpósio Brasileiro de Informática na Educação - SBIE 2014 Dourados, MS: Sociedade Brasileira de Computação - SBC, 2014. Disponível em: <http://www.br-ie.org/pub/index.php/sbie/issue/view/87>. Acesso em: 23 jun. 2017.

V CONGRESSO BRASILEIRO DE INFORMÁTICA NA EDUCAÇÃO - CBIE, 2016, Uberlândia, MG. $2^{\circ}$ Simpósio Brasileiro de Informática na Educação - SBIE 2016. Uberlândia, MG: Sociedade Brasileira de Computação - SBC, 2016. Disponível em: <http://www.br-ie.org/pub/index.php/sbie/issue/view/155>. Acesso em: 23 jun. 2017.

INFORMÁTICA NA EDUCAÇÃO: TEORIA \& PRÁTICA: TECNOLOGIA ASSISTIVA, PROCESSO ENSINO-APRENDIZAGEM E ESTÉTICA. Porto Alegre, RS: Universidade Federal do Rio Grande do Sul - UFRGS, v. 19, n. 1, set. 2016. Quadrimestral. Disponível em: <http://zip.net/bdtLYG>. Acesso em: 23 jun. 2017.

INFORMÁTICA NA EDUCAÇÃO: TEORIA \& PRÁTICA: EDUCAÇÃO, INCLUSÃO E AMBIENTE VIRTUAL DE APRENDIZAGEM. Porto Alegre, RS: Universidade Federal do Rio Grande do Sul - UFRGS, v. 18, n. 1, set. 2015. Quadrimestral. Disponível em: <http://zip.net/bktLsw>. Acesso em: 23 jun. 2017.

REVISTA NOVAS TECNOLOGIAS NA EDUCAÇÃO (RENOTE). Porto Alegre, RS: Centro Interdisciplinar de Novas Tecnologias na Educação - Cinted, v. 10, n. 1, 2012. Semestral. Disponível em: <http://zip.net/bjtLn1>. Acesso em: 23 jun. 2017.

REVISTA NOVAS TECNOLOGIAS NA EDUCAÇÃO (RENOTE). Porto Alegre, RS: Centro Interdisciplinar de Novas Tecnologias na Educação - Cinted, v. 11, n. 3, 2013. Trimestral. Disponível em: 〈http://zip.net/bwtKYw>. Acesso em: 23 jun. 2017. 
REVISTA NOVAS TECNOLOGIAS NA EDUCAÇÃO (RENOTE). Porto Alegre, RS: Centro Interdisciplinar de Novas Tecnologias na Educação - Cinted, v. 12, n. 2, 2014. Trimestral. Disponível em: 〈http://zip.net/bytLWs〉. Acesso em: 23 jun. 2017.

REVISTA NOVAS TECNOLOGIAS NA EDUCAÇÃO (RENOTE). Porto Alegre, RS: Centro Interdisciplinar de Novas Tecnologias na Educação - Cinted, v. 14, n. 2, 2016. Semestral. Disponível em: 〈http://zip.net/bctLmK〉. Acesso em: 23 jun. 2017.

REVISTA NOVAS TECNOLOGIAS NA EDUCAÇÃO (RENOTE). Porto Alegre, RS: Centro Interdisciplinar de Novas Tecnologias na Educação - Cinted, v. 14, n. 1, 2016. Trimestral. Disponível em: <http://zip.net/bttL84>. Acesso em: 23 jun. 2017.

REVISTA BRASILEIRA DE INFORMÁTICA NA EDUCAÇÃO (RBIE). Porto Alegre, RS: Comissão Especial de Informática na Educação - CEIE, v. 22, n. 3, 2014. Disponível em: <http://www.br-ie.org/pub/index.php/rbie/issue/view/85>. Acesso em: 23 jun. 2017.

REVISTA BRASILEIRA DE INFORMÁTICA NA EDUCAÇÃO (RBIE). Porto Alegre, RS: Comissão Especial de Informática na Educação - CEIE, v. 23, n. 1, 2015. Disponível em: <http://www.br-ie.org/pub/index.php/rbie/issue/view/94>. Acesso em: 23 jun. 2017.

REVISTA BRASILEIRA DE INFORMÁTICA NA EDUCAÇÃO (RBIE). Porto Alegre, RS: Comissão Especial de Informática na Educação - CEIE, v. 24, n. 3, 2016. Disponível em: <http://www.br-ie.org/pub/index.php/rbie/issue/view/160>. Acesso em: 23 jun. 2017.

REVISTA BRASILEIRA DE INFORMÁTICA NA EDUCAÇÃO (RBIE). Porto Alegre, RS: Comissão Especial de Informática na Educação - CEIE, v. 24, n. 2, 2016. Disponível em: <http://www.br-ie.org/pub/index.php/rbie/issue/view/159>. Acesso em: 23 jun. 2017. 